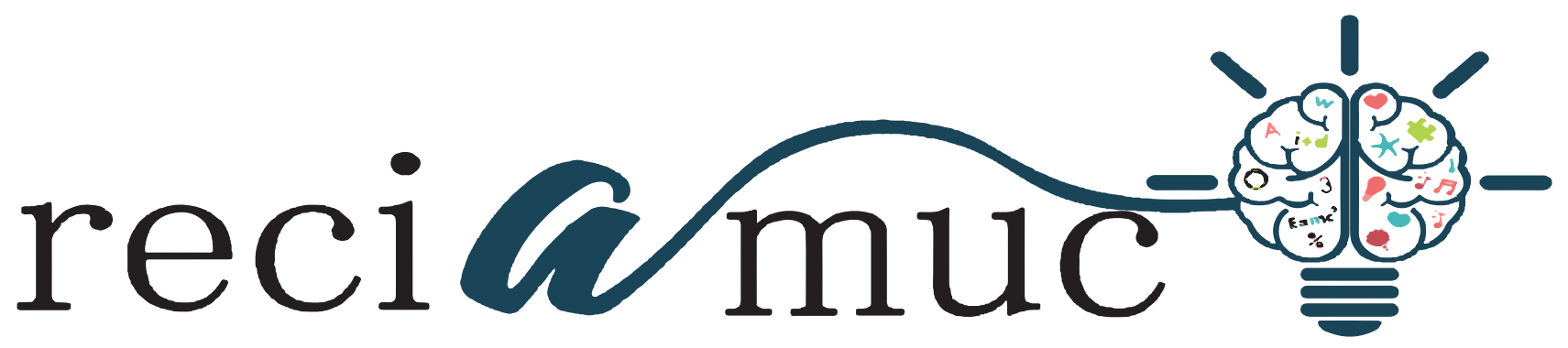

DOl: $10.26820 /$ reciamuc/4.(4).noviembre.2020.263-272

URL: https://reciamuc.com/index.php/RECIAMUC/article/view/584

EDITORIAL: Saberes del Conocimiento

REVISTA: RECIAMUC

ISSN: 2588-0748

TIPO DE INVESTIGACIÓN: Artículo de revisión

Código UNESCO: 32 Ciencias Médicas

PAGINAS: 263-272

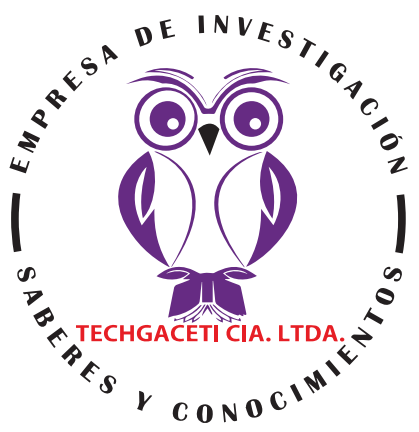

\title{
Sintomatología y tratamiento en cada etapa del paciente con cáncer de esófago
}

Symptomatology and treatment at each stage of the
esophageal cancer patient

Sintomatologia e tratamento em cada fase do doente com cancro esofágico

Fressia Carolina Zambrano Zambrano'; María Agustina Vélez Macías²; Nelson Ricardo Chacha Suscal3; Gema Gabriela Basurto Macias"; Fredy Andrés Pesantez Durán ${ }^{5}$

RECIBIDO: 18/08/2020 ACEPTADO: 20/09/2020 PUBLICADO: 12/11/2020

1. Médico Cirujano; Egresada en Master Universitario en Dirección y Gestión Sanitaria; Centro de Salud Plan de Vivienda Santo Domingo; Ecuador; fressia2312@gmail.com; https://orcid.org/0000-0003-4226-1156

2. Médico Cirujano; Cursando Maestría de Gerencia en Salud; Hospital Oncológico de SOLCA; Portoviejo, Ecuador; joseyagus001@gmail. com; https://orcid.org/0000-0003-3137-7006

3. Médico General; Médico Residente; Hospital Vicente Corral Moscoso; Cuenca, Ecuador; ricardocsmed93@gmail.com; https://orcid. org/0000-0002-3849-4806

4. Médico Cirujano; Médico General en Funciones Hospitalarias; Hospital Dr Gustavo Domínguez Zambrano; Santo Domingo, Ecuador; gemy_19_06_gb@hotmail.com; https://orcid.org/0000-0002-9801-806X

5. Médico; Médico Residente; Hospital Vicente Corral Moscoso; Cuenca, Ecuador; fredy_pesantez@hotmail.com; https://orcid.org/00000002-9735-6091

CORRESPONDENCIA

Fressia Carolina Zambrano Zambrano

fressia2312@gmail.com

Santo Domingo; Ecuador

() RECIAMUC; Editorial Saberes del Conocimiento, 2020 


\section{RESUMEN}

El cáncer es uno de los problemas de salud más graves del mundo. Es la segunda causa principal de muerte en las Américas. Entre los tipos de cáncer, el cáncer de esófago es el octavo más frecuente en todo el mundo y ocupa el tercer lugar entre los de origen gastrointestinal. El cáncer de esófago es un tumor que se forma en los tejidos que revisten el esófago, el tubo muscular a través del cual los alimentos pasan desde la garganta al estómago. A lo largo del tiempo, el tratamiento ha mejorado significativamente, como consecuencia las tasas de supervivencia igualmente han mejorado. El objetivo del presente estudio consiste en plasmar la sintomatología básica del paciente con cáncer de esófago, así como las recomendaciones para su tratamiento según la etapa de su enfermedad. El modelo de investigación es una revisión de tipo documental bibliográfico. La sintomatología suele presentarse en etapas más avanzadas de la enfermedad. Entre los síntomas más frecuentes, casi universal, se encuentra la disfagia. En cuanto a la estadificación, los estadios del cáncer de esófago se indican con números romanos que van del 0 al IV. Es importante la estadificación por cuanto, entre los aspectos a considerar para la elección del mejor tratamiento, se encuentra el estadio del cáncer. El tratamiento estándar consiste en cirugía, radioterapia, quimioterapia, quimiorradioterapia, terapia láser y electrocoagulación, entre otros. El tratamiento en base a la estadificación del cáncer de esófago permite adaptar la terapéutica a las necesidades específicas que permitan mejorar el pronóstico del paciente, combinando varias técnicas, de ser necesario, y considerando las complicaciones que puedan presentarse con la finalidad de prevenirlas, proporcionando la mejor calidad de vida al paciente en base a la etapa del cáncer de esófago en que se encuentre.

Palabras clave: Sintomatología, Cáncer de esófago, Tratamiento, Estadificación, Paciente oncológico.

\section{ABSTRACT}

Cancer is one of the most serious health problems in the world. It is the second leading cause of death in the Americas. Among the types of cancer, esophageal cancer is the eighth most common in the world and ranks third among those of gastrointestinal origin. Esophageal cancer is a tumor that forms in the tissues that line the esophagus, the muscular tube through which food passes from the throat to the stomach. Over time, treatment has improved significantly, as a consequence survival rates have also improved. The objective of this study is to capture the basic symptoms of the patient with esophageal cancer, as well as the recommendations for its treatment according to the stage of the disease. The research model is a bibliographic documentary type review. Symptoms usually appear in more advanced stages of the disease. Among the most frequent symptoms, almost universal, is dysphagia. As for staging, the stages of esophageal cancer are indicated by Roman numerals ranging from 0 to IV. Staging is important because, among the aspects to consider when choosing the best one, is the stage of the cancer. Standard treatment consists of surgery, radiation therapy, chemotherapy, chemoradiotherapy, laser therapy, and electrocoagulation, among others. The treatment based on the staging of esophageal cancer allows to adapt the therapy to the specific needs that will improve the patient's prognosis, combining several techniques, if necessary, and consider the complications that may occur in order to prevent them, make the better quality of life for the patient based on the stage of esophageal cancer.

Keywords: Symptomatology, Esophageal cancer, Treatment, Staging, Oncological patient.

\section{RESUMO}

O cancro é um dos problemas de saúde mais graves do mundo. É a segunda principal causa de morte nas Américas. Entre os tipos de cancro, o cancro do esófago é o oitavo mais comum no mundo e ocupa o terceiro lugar entre os de origem gastrointestinal. O cancro esofágico é um tumor que se forma nos tecidos que revestem o esófago, o tubo muscular através do qual os alimentos passam da garganta para o estômago. Ao longo do tempo, o tratamento melhorou significativamente, como consequência, as taxas de sobrevivência também melhoraram. O objectivo deste estudo é captar os sintomas básicos do paciente com cancro do esófago, bem como as recomendações para o seu tratamento de acordo com a fase da doença. O modelo de investigação é uma revisão bibliográfica de tipo documental. Os sintomas aparecem geralmente em fases mais avançadas da doença. Entre os sintomas mais frequentes, quase universais, encontra-se a disfagia. Quanto ao estadiamento, as fases do cancro do esófago são indicadas por numerais romanos que vão de 0 a IV. O estadiamento é importante porque, entre os aspectos a considerar na escolha do melhor, está a fase do cancro. O tratamento padrão consiste em cirurgia, radioterapia, quimioterapia, quimioradioterapia, laserterapia, e electrocoagulação, entre outros. 0 tratamento baseado na fase do cancro do esófago permite adaptar a terapia às necessidades específicas que irão meIhorar o prognóstico do paciente, combinando várias técnicas, se necessário, e considerar as complicações que podem ocorrer a fim de as prevenir, fazer a melhor qualidade de vida para o paciente com base na fase do cancro do esófago.

Palavras-chave: Sintomatologia, Cancro esofágico, Tratamento, Estadiamento, Paciente oncológico. 


\section{Introducción}

El cáncer es uno de los problemas de salud más graves del mundo. "Es la segunda causa principal de muerte en las Américas. En el 2018, se diagnosticaron unos 3,8 millones de casos y 1,4 millones de personas murieron por esta enfermedad". (Organización Panamericana de la Salud - OPS, 2020)

En tal sentido, Encinas, Corral, Fernández, Ruano, \& Álvarez, (2016) refieren que de todos los tipos de cáncer, el cáncer de esófago "es el octavo más frecuente en todo el mundo y ocupa el tercer lugar entre los de origen gastrointestinal". (p. 353)

El cáncer de esófago es un tumor que se forma en los tejidos que revisten el esófago, el tubo muscular a través del cual los alimentos pasan desde la garganta al estómago. Los dos principales tipos de cáncer de esófago son el carcinoma de células escamosas (carcinoma escamocelular) y el adenocarcinoma. El primero comienza en las células planas que forman el revestimiento interno del esófago, mientras que el adenocarcinoma comienza en las células que producen y liberan moco y otros líquidos. La frecuencia de ambos tipos es la misma. (Fundación Contra el Cáncer, 2012)

Está situado entre los diez cánceres que se presentan con más frecuencia en el mundo. Su incidencia presenta grandes variaciones geográficas. entre las áreas considerándose de alta frecuencia se encuentran Asia y África Central y del Sur, con más de 100 casos por 100.000 habitantes/año. En el caso de Europa las tasas más altas de incidencia se presentan en los siguientes países: Rusia, Francia, Reino Unido e Irlanda. España se ubica, con respecto al resto de los países de Europa, en un término medio de incidencia, se estima que aproximadamente $8 / 100.000$ hombres y $1 / 100.000$ mujeres. El cáncer de esófago se considera de mayor frecuencia en el hombre que en la mujer, pudiendo oscilar la relación entre 3 y 10 hombres por cada mujer, dependiendo del área geográfica. "La edad habitual de presentación es entre los 55 y los 70 años, siendo infrecuentes los casos en personas por debajo de los 40 años". (Gallego Plazas, 2020)

El pronóstico de esta enfermedad va a depender, en gran parte, del estadio en que se encuentra, sin embargo, en términos generales es malo. Presenta una supervivencia a 5 años: < 5\%, esto como consecuencia de que muchos pacientes se presentan con un estado de enfermedad avanzada. Aquellos pacientes con cáncer limitado a la mucosa llegan a tener una tasa de supervivencia de alrededor del $80 \%$, que desciende a $<50 \%$ en los casos de compromiso submucoso, hasta un $20 \%$ con la extensión a la muscular propia, a un 7\% con la extensión a estructuras adyacentes y a $<3 \%$ con metástasis a distancia. (Nguyen, 2019)

A lo largo del tiempo, el tratamiento ha mejorado significativamente, como consecuencia las tasas de supervivencia igualmente han mejorado." Durante los años sesenta y setenta, solo alrededor de $5 \%$ de los pacientes sobrevivía al menos 5 años después del diagnóstico. Actualmente, alrededor de $20 \%$ de los pacientes sobrevive al menos 5 años después del diagnóstico". Tomando en consideración a los pacientes con todas las etapas de cáncer de esófago. Las tasas de supervivencia para personas con cáncer en etapa inicial son aún mucho mayores. (Sociedad Americana Contra el Cáncer, 2021)

En virtud de lo anterior, el conocimiento que se pueda tener tanto de la sintomatología de la enfermedad, así como del tratamiento, resultan de vital importancia para un diagnóstico lo más tempranamente posible y la decisión de un plan terapéutico ajustado a las necesidades y preferencias del paciente, esto con la finalidad de mejorar el pronóstico, evitar complicaciones y reducir la tasa de supervivencia de este tipo de cáncer.

El objetivo del presente estudio consiste en plasmar la sintomatología básica del pa-

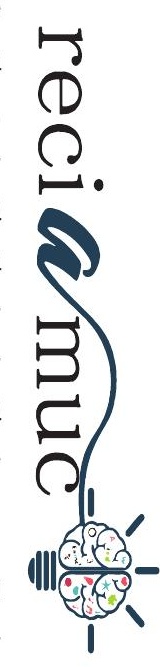


ciente con cáncer de esófago, así como las recomendaciones para su tratamiento según la etapa de su enfermedad. Para lo cual es importante abarcar aspectos como la estadificación básica del cáncer de esófago y el tratamiento general.

\section{Materiales y métodos}

Para el desarrollo del presente estudio fueron ubicados una serie de archivos digitalizados, cuya búsqueda de contenidos científicos se llevó a cabo durante el mes de enero de 2021. Dicha metodología lo ubica como un estudio de tipo documental bibliográfico.

Se formularon ecuaciones de búsqueda, con palabras clave, operadores lógicos y booleanos, en determinadas bases de datos y/o buscadores especializados, tales como: SciELO, Researchgate, entre otros. Asimismo, se usaron páginas web relacionadas con el área de la salud, con amplio reconocimiento científico a nivel nacional e internacional, entre las que destacamos: Organización Mundial de la Salud (OMS), Organización Panamericana de la Salud (OPS), Sociedad Española de Oncología Médica (SEOM), Manuales MSD, Clínica Mayo, Instituto Nacional del Cáncer de los Estados Unidos, Fundación Contra el Cáncer, Sociedad Americana Contra el Cáncer, entre otras.

Algunas de las formulaciones y descriptores utilizados con los que se obtuvieron mejores resultados fueron los siguientes: "cáncer de esófago"; "etapas del cáncer de esófago"; "síntomas del cáncer de esófago", "tratamiento del cáncer de esófago" y "tratamiento por etapas del cáncer de esófago". La información obtenida fue filtrada bajo los criterios de idioma (español); disponibilidad del contenido (completo); periodo de publicación (2012-2021), tipo de estudio: revisiones sistemáticas, de cohorte y de casos o de controles, informe de casos; y, clase de material bibliográfico (consensos, manuales, ensayos, tesis de grado, posgrado o doctorado, informes y otras cla- ses de contenidos).

Asimismo, se seleccionaron los recursos antes mencionados en base a su producción, aval o promoción por parte de instituciones, entes, organizaciones, sociedades o asociaciones de profesionales en el área de la salud, de carácter público o privado, nacionales, internacionales o multilaterales con reconocimiento científico dentro de la comunidad de la salud.

Por último, fueron desestimados aquellos contenidos repetidos (duplicados), editoriales, anotaciones académicas y otros tipos de materiales bibliográficos de escaso valor científico, con bajo nivel de evidencia o aportado por tratadistas sin acreditación en el área de la salud o medicina.

\section{Resultados}

\section{Sintomatología}

El cáncer de esófago en estadios tempranos tiende a ser asintomático, esta es la principal causa por la cual es tan difícil diagnosticarlo en etapas tempranas. Cuando la luz del esófago se estrecha hasta alrededor de $<14 \mathrm{~mm}$, suele aparecer uno de sus síntomas más característicos: la disfagia. "Primero, el paciente tiene dificultad para tragar alimentos sólidos, después semisólidos, por último, alimentos líquidos y saliva. Esta progresión sostenida sugiere un proceso maligno en crecimiento más que un espasmo, un anillo benigno o una estenosis péptica". Otro síntoma es el dolor torácico, el cual puede irradiar a la espalda. Asimismo, se suele presentar pérdida de peso, pese a que el paciente mantenga un buen apetito. Se puede presentar parálisis de las cuerdas vocales y ronquera, debido a la compresión del nervio laríngeo recurrente. Esta compresión también puede ocasionar dolor espinal, hipo o parálisis del diafragma. Otro síntoma que se puede presentar es la disnea como consecuencia de los derrames pleurales malignos o las metástasis pulmonares. Por otro lado, puede presentarse odinofagia, vómitos, hematemesis, 
melena, anemia ferropénica, aspiración y tos, por causa del compromiso tumoral intraluminal. Las fístulas entre el esófago y el árbol traqueobronquial pueden causar un absceso pulmonar y una neumonía. Otros hallazgos pueden ser un síndrome de la vena cava superior, ascitis maligna y dolor óseo. (Nguyen, 2019)

Asimismo, pueden presentarse algunos síntomas respiratorios como "tos e infecciones respiratorias. Suele ser derivado de la afectación del árbol respiratorio secundaria al tumor esofágico. Si aparece en un paciente con cáncer de esófago suele asociarse a un tumor avanzado". (Gallego Plazas, 2020)

\section{Estadificación del cáncer de esófago}

Las decisiones terapéuticas para el cáncer de esófago dependen de varias consideraciones, entre las que se encuentran: la estadificación del tumor, su tamaño y localización, el estado de salud general del paciente y sus deseos o preferencias (muchos eligen renunciar al tratamiento intensivo). (Nguyen, 2019)

Posterior al diagnóstico, el médico tratante debe valorar si el cáncer se ha propagado y si es así, a qué distancia. Este proceso se llama estadificación (o determinación de la etapa). "La etapa (estadio) de un cáncer describe cuánto cáncer hay en el cuerpo, y ayuda a determinar qué tan grave es el cáncer, así como la mejor manera de tratarIo". (Sociedad Americana Contra el Cáncer, 2020)

Los estadios del cáncer de esófago se indican con números romanos que van del 0 al IV, y los estadios más bajos indican que el cáncer es pequeño y afecta solo las capas superficiales del esófago. En el estadio IV, el cáncer se considera avanzado y se ha propagado a otras zonas del cuerpo. (Clínica Mayo, 2020)

Para una mejor comprensión, en la siguiente clasificación por estadios se han agrupado las histologías epidermoide y adenocar- cinoma, así como los diferentes grados de diferenciación, por lo que la clasificación aquí descrita resulta una simplificación de la actualmente establecida:

- Estadio 0: también denominado carcinoma in situ. El cáncer se localiza sólo en la parte más superficial de la mucosa esofágica, sin sobrepasarla.

- Estadio I: Tumores bien diferenciados, sin infiltración ganglionar locorregional por metástasis, que alcanzan como máximo la capa muscular propia sin sobrepasarla.

- Estadio II: El tumor no alcanza la capa adventicia, asociando un máximo de dos ganglios linfáticos locorregionales infiltrados por metástasis; o alcanza sin sobrepasar la capa adventicia sin presentar ganglios linfáticos con metástasis.

- Estadio III: El tumor afecta a la capa adventicia, asociando un máximo de 2 ganglios linfáticos locorregionales infiltrados por metástasis; o presenta afectación ganglionar en más de 2 y menos de 7 ganglios, independientemente de la profundidad de invasión del tumor sin que éste afecte a estructuras adyacentes.

- Estadio IV: El tumor compromete a estructuras adyacentes, o presenta una importante afectación locorregional ganglionar (siete o más ganglios afectos), o se ha extendido a distancia (a otros órganos del cuerpo). (Gallego Plazas, 2020)

\section{Tratamiento estándar}

Referente al tratamiento, Téllez \& Hodelín, (2016) citando al National Cancer Institute of EE. UU, menciona los tipos de tratamiento estándar para el cáncer de esófago de la siguiente manera:

- Cirugía: es el tratamiento más común para el cáncer de esófago. Parte del 
esófago se puede extirpar en una operación que se llama esofagectomía.

- Radioterapia: se usan rayos X de alta energía u otros tipos de radiación para destruir células cancerosas o impedir que crezcan. Hay dos tipos de radioterapia:

- Radioterapia externa: se usa una máquina afuera del cuerpo que envía la radiación hacia el cáncer.

- Radioterapia interna: se usa una sustancia radiactiva sellada en agujas, semillas, cables o catéteres, que se coloca directamente en el cáncer o cerca del mismo.

- Quimioterapia: se usan medicamentos para interrumpir el crecimiento de células cancerosas, ya sea mediante su destrucción o impidiendo su multiplicación. Cuando la quimioterapia se administra por boca o se inyecta en una vena o músculo, los medicamentos ingresan en el torrente sanguíneo y pueden llegar a las células cancerosas de todo el cuerpo (quimioterapia sistémica). Cuando la quimioterapia se coloca directamente en el líquido cefalorraquídeo, un órgano o una cavidad corporal como el abdomen, los medicamentos afectan principalmente las células cancerosas de esas áreas (quimioterapia regional).

- Quimiorradioterapia: combina la quimioterapia y la radioterapia para aumentar los efectos de ambas.

- Terapia láser: se usa un haz de rayo láser (haz angosto de luz intensa) para destruir células cancerosas.

- Electrocoagulación: es el uso de una corriente eléctrica para destruir células cancerosas. (p. 11, 12)

\section{El tratamiento según la etapa}

\section{Etapa 0 o In situ}

Braghetto et al., (2016) sugieren las siguien- tes opciones para el tratamiento del cáncer de esófago In Situ:

Se han sugerido diferentes opciones de terapia endoscópica como el uso de terapia fotodinámica o ablación con radiofrecuencia que se está utilizando especialmente en paciente con displasia de alto grado de localización mucosa, en la que en Oriente se utiliza más frecuentemente la resección submucosa endoscópica. El problema de estas técnicas es que pueden presentar recurrencia local entre 15 a 30\%, recurrencia metacrónica en más de un $20 \%$, pueden retrasar una cirugía, por lo que el seguimiento en este caso debe ser muy acucioso tanto con endoscopia, biopsias y endosonografía para detección de recurrencia precoz. La sobrevida a 5 años es de 84\% libre de tumor. En el cáncer escamoso que compromete la muscular de la mucosa la incidencia de compromiso linfonodal es de un 6-18\%, por lo que la mucosectomía estaría indicada sólo en tumores que comprometen hasta la mucosa superficial (m1), escamosos < $3 \mathrm{~cm}$ que comprometen menos de $3 / 4$ de la circunferencia esofágica. En el adenocarcinoma, el $10 \%$ de los tumores que comprometen la muscular de la mucosa presentan compromiso linfonodal, por lo que también estaría indicada la mucosectomía en la displasia de alto grado o tumores que comprometen sólo la mucosa superficial15. Sin embargo, es posible que hasta un 30\% de las displasia de alto grado pueden presentar un cáncer invasor más allá de la muscular de la mucosa y que más del 10\% presente recurrencia de la enfermedad, por lo que estos pacientes deben quedar en estricto seguimiento endoscópico. Debido a la gran morbimortalidad de la esofaguectomía (> $5 \%$ ), esta última no estaría indicada en esta etapa de la enfermedad. (p. 95, 96)

\section{Estadio I}

Para este estadio temprano del cáncer de esófago se pueden considerar las siguientes opciones de tratamiento: 
Está reportado que, en el carcinoma mucoso superficial, independiente del tipo histológico, la incidencia de compromiso linfonodal es un $0-5 \%$, por lo que se ha planteado la mucosectomía endoscópica en este estadio tumoral. Por el contrario, en los que comprometen la muscular de la mucosa, la incidencia de compromiso linfonodal es mayor al 5\%, por lo que estaría indicada la esofaguectomía. Según las guías del NCCN, los tumores que comprometen hasta la muscular de la mucosa pueden ser tratados mediante esofaguectomía o mucosectomía, esta última siempre cuando no tengan compromiso linfonodal, no tengan invasión vascular ni linfática, ni sean mal diferenciados. Otra indicación es cuando se presenta en pacientes que no están en condiciones de ser operados por sus patologías asociadas. Si bien hay estudios que demuestran buena sobrevida a 5 años mediante mucosectomía (80\% aproximadamente), con cirugía la sobrevida puede alcanzar un $100 \%$ a 5 años. Por otra parte, es muy difícil saber cuándo efectivamente un cáncer compromete sólo la mucosa superficial, ya que la endosonografía no presenta una exactitud diagnóstica de un 100\%. En los cánceres con compromiso de la muscular de la mucosa y submucosos la incidencia de compromiso linfonodal es mayor a un 30\% por lo que en estos casos la mucosectomía no estaría indicada1. En el consenso de la Sociedad Latinoamericana de Gastroenterología Oncológica del 2011, al consultarle a los panelistas sobre si para el estadio I el tratamiento de elección era la resección endoscópica o la esofaguectomía, el 54\% votó por la resección quirúrgica. En la mesa de discusión se consensuó que la decisión debe ser dada caso a caso dependiendo de los resultados de los estudios y características generales del paciente. Los tipos de esofaguectomía actualmente en uso son la esofaguectomía transhiatal, la misma técnica, pero con preservación del vago ("vagal Sparing") y la esofaguectomía en block, que son técnicas poco difundidas y poco usadas. Actualmente las técnicas mini invasi- vas vía toracoscópica y laparoscópicas han ganado mucha difusión y muchos grandes centros de excelencia han adoptado esta técnica como la de elección. (Braghetto et al., 2016, p. 96)

\section{Estadio II}

Esta etapa incluye "cánceres que han crecido hacia la capa muscular principal del esófago o hacia el tejido conectivo en el exterior del esófago. Asimismo, incluye algunos cánceres que se han propagado a 1 o 2 ganglios linfáticos cercanos". (Sociedad Americana Contra el Cáncer, 2020)

Según el Instituto Nacional del Cáncer de los Estados Unidos, (2020) las opciones de tratamiento estándar del cáncer de esófago en estadio II son las siguientes:

- Quimiorradioterapia seguida de cirugía: En ensayos de fase III se comparó la quimiorradioterapia preoperatoria simultánea y la cirugía sola para pacientes de cáncer de esófago.

- Cirugía sola: La tasa de supervivencia de los pacientes con cáncer de esófago es precaria. El tratamiento quirúrgico del cáncer de esófago resecable produce tasas de supervivencia a 5 años de 5 a $30 \%$, la supervivencia es más alta en los pacientes con cáncer en estadio temprano. Los tumores pequeños asintomáticos que están limitados a la mucosa o submucosa esofágica son hallazgos fortuitos. La cirugía es el tratamiento preferido para estos tumores pequeños. Una vez hay síntomas (por ejemplo, disfagia, en la mayoría de los casos), por lo general el cáncer de esófago ya invadió o traspasó la capa muscular propia, y es posible que se encuentren metástasis ganglionares o en otros órganos.

- Quimioterapia seguida de cirugía: Los efectos de la quimioterapia preoperatoria están en evaluación en ensayos aleatorizados. En varios estudios se demostró un beneficio para la supervivencia de 
la quimioterapia preoperatoria comparada con la cirugía sola. Sin embargo, en un estudio aleatorizado grande no se pudo confirmar un beneficio para la supervivencia de la quimioterapia preoperatoria.

- Quimiorradioterapia definitiva.: Para los pacientes con contraindicaciones médicas para la cirugía o que tienen tumores irresecables, la eficacia de la quimiorradioterapia definitiva se estableció en numerosos ensayos controlados aleatorizados. Para los pacientes con carcinoma de células escamosas de esófago, la quimiorradioterapia definitiva quizás produzca desenlaces equivalentes en comparación con la quimiorradiación preoperatoria seguida de resección quirúrgica.

\section{Estadio III}

Para las personas que están lo suficientemente saludables. Es importante considerar otros aspectos y adaptar el tratamiento en base a cada uno de ellos, de la siguiente manera:

El tratamiento para estos cánceres frecuentemente consiste en quimiorradiación seguida de cirugía. Los pacientes con adenocarcinoma en el lugar donde el estómago y el esófago se unen (la unión gastroesofágica) a veces se tratan con quimioterapia (sin radiación) seguida de cirugía. La cirugía solamente podría ser una opción para algunos tumores pequeños. Si la cirugía es el primer tratamiento, se puede recomendar después quimiorradiación, especialmente si el cáncer es un adenocarcinoma o si existen signos de que haya quedado algo de cáncer. En algunos casos, (especialmente para los cánceres que se encuentran en la parte superior del esófago), se pudiera recomendar quimiorradiación como tratamiento principal en lugar de cirugía. Los pacientes que no se someten a cirugía necesitan seguimiento minucioso con endoscopia para detectar posibles signos de cáncer remanente. Desafortunadamente, aun cuando el cáncer no se puede ver, puede estar presente debajo del revestimiento interno del esófago. Por lo tanto, el seguimiento minucioso es muy importante. Los pacientes que no se pueden someter a cirugía debido a que presentan otros problemas de salud graves usualmente pueden ser tratados con quimiorradiación. (Sociedad Americana Contra el Cáncer, 2020)

\section{Estadio IV o etapa avanzada}

El objetivo del tratamiento en esta etapa básicamente es la paliación, que consiste en el alivio de síntomas con mejoría en la calidad de vida. Asimismo, se trata de prolongar la supervivencia del paciente tanto como sea posible. El pilar fundamental del tratamiento en este estadio es la quimioterapia. "Estudios recientes han abierto la oportunidad para el uso de la inmnunoterapia en líneas sucesivas de tratamiento, tras fracaso de la quimioterapia de primera línea, en tumores epidermoides y/o con especial susceptibilidad a este tratamiento". Con el propósito de mejorar la sintomatología del paciente: dolor, imposibilidad para la ingesta de alimentos, entre otros, puede indicarse el uso de radioterapia externa, radioterapia interna, terapia láser, prótesis esofágicas, y cirugía paliativa, según sea el caso. (Gallego Plazas, 2020)

Por otra parte, la Fundación Contra el Cáncer (2012) refiere al respecto que, para los pacientes con cáncer de esófago metastásico pueden considerarse diferentes opciones de tratamiento para aliviar sus síntomas. La elección que se realice dependerá de su situación en particular. Para ello recomienda:

- Tratamiento local: Braquiterapia es un tipo de radioterapia en el que se coloca un material radioactivo directamente en el tumor o cerca de él. Debido a la localización cercana con respecto al tumor y a la corta distancia que debe viajar la radiación, pueden administrarse dosis de radiación más elevadas que en la radioterapia externa, que usa radiación 
procedente del exterior del cuerpo y dirigida a la zona del tumor. Esta estrategia puede aliviar las molestias y dificultades para la deglución (para tragar) en los pacientes con cáncer metastásico de esófago, y ha demostrado producir mejores efectos a largo plazo y menos efectos nocivos que la colocación de un stent. Un stent es un tubo metálico colocado en el interior del esófago para impedir que sea bloqueado por el crecimiento del tumor y, con ello, permitir el paso de los alimentos. Los efectos secundarios de la braquiterapia son dolor pasajero de garganta y mareos. Con un stent, se corre el riesgo de que el tumor crezca cubriendo un extremo del mismo $y$, tras algún tiempo, vuelva a bloquear el esófago.

- Tratamiento sistémico: Un tratamiento sistémico tiene el objetivo de actuar en todas las células cancerosas en cualquier parte del cuerpo. La quimioterapia es el principal tipo de tratamiento sistémico. En contraposición al tratamiento local, como la cirugía o la radioterapia, que actúa sobre las células cancerosas en una zona definida. La quimioterapia puede contribuir a reducir los síntomas y debe tenerse en cuenta especialmente en aquellos pacientes en buen estado de salud y general. Solían usarse cisplatino y 5-fluorouracilo. Sin embargo, algunos fármacos más nuevos de las mismas clases de medicamentos parecen ofrecer una mejor eficacia y mejor calidad de vida. (p. 19)

\section{Conclusiones}

El cáncer de esófago es uno de los diez cánceres más importantes en todo el mundo y, por tanto, un problema de salud mundial que requiere especial atención. Su característica asintomática, al inicio de la enfermedad ha sido uno de los principales obstáculos en el diagnóstico temprano.

La sintomatología suele presentarse en etapas más avanzadas de la enfermedad.
Entre los síntomas más frecuentes, casi universal, se encuentra la disfagia. En cuanto a la estadificación, los estadios del cáncer de esófago se indican con números romanos que van del 0 al IV. Los estadios más bajos indican que el cáncer es pequeño, mientras que en los más altos se considera el cáncer avanzado y propagado a otras zonas del cuerpo.

Es importante la estadificación por cuanto, entre los aspectos a considerar para la elección del mejor tratamiento, se encuentra el estadio del cáncer. El tratamiento estándar consiste en cirugía, radioterapia, quimioterapia, quimiorradioterapia, terapia láser y electrocoagulación, entre otros.

El tratamiento en base a la estadificación del cáncer de esófago permite adaptar la terapéutica a las necesidades específicas que permitan mejorar el pronóstico del paciente, combinando varias técnicas, de ser necesario, y considerando las complicaciones que puedan presentarse con la finalidad de prevenirlas, proporcionando la mejor calidad de vida al paciente en base a la etapa del cáncer de esófago en que se encuentre.

\section{Bibliografía}

Braghetto, I., Cardemil, G., Csendes, A., Lanzarini, E., Mushle, M., Venturelli, F., . . Gattini, F. (2016). Resultados de la cirugía actual para el tratamiento de cáncer de esófago. Revista Chilena Cirugía, 68(1), 94-106. Recuperado el 20 de Enero de 2021, de https://www.researchgate.net/profile/ Italo_Braghetto/publication/301714268_RESULTADOS_DE_LA_CIRUGIA_ACTUAL_PARA_EL_ TRATAMIENTO_DEL_CANCER_DE_ESOFAGO/ links/5745ae2508ae9ace8424016d/RESULTADOS-DE-LA-CIRUGIA-ACTUAL-PARA-EL-TRATAMIENTO-DEL-CANCER-DE-ESOFAGO.p

Clínica Mayo. (04 de Abril de 2020). Clínica Mayo. Recuperado el 08 de Enero de 2021, de https:// www.mayoclinic.org/es-es/diseases-conditions/esophageal-cancer/diagnosis-treatment/ drc-20356090\#: : text=Una\%20de\%20las\%20 operaciones \%20utilizadas,y\%20los\%20ganglios\%20linf\%C3\%A1ticos\%20cercanos.

Encinas, J., Corral, M., Fernández, G., Ruano, R., \& 


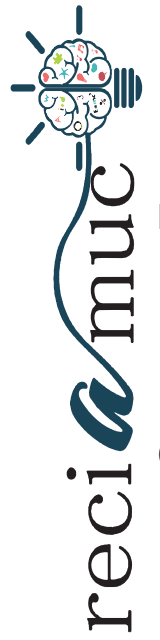

Álvarez, A. (2016). Cáncer de esófago: particularidades anatómicas, estadificación y técnicas de imágenes. Radiología, 58(5), 352-365. doi:http:// dx.doi.org/10.1016/j.rx.2016.06.004

Fundación Contra el Cáncer. (2012). Sociedad Europea de Medicina Ondológica (ESMO). Recuperado el 13 de Enero de 2021, de https://www.esmo. org/content/download/6607/115057/file/ES-Cancer-de-Esofago-Guia-para-Pacientes.pdf

Gallego Plazas, J. (28 de Enero de 2020). Sociedad Española de oncología Médica (SEOM). Recuperado el 10 de Enero de 2021, de https://seom.org/ info-sobre-el-cancer/esofago?showall=1

Instituto Nacional del Cáncer de los Estados Unidos. (20 de Agosto de 2020). Instituto Nacional del Cáncer de los Estados Unidos. Recuperado el 25 de Enero de 2021, de https://www.cancer. gov/espanol/tipos/esofago/pro/tratamiento-esofago-pdq\#_218_toc

Nguyen, M. (Junio de 2019). Manuales MSD. Recuperado el 03 de Enero de 2021, de https://www. msdmanuals.com/es-ve/professional/trastornos-gastrointestinales/tumores-del-aparato-digestivo/c\%C3\%A1ncer-de-es\%C3\%B3fago
Organización Panamericana de la Salud - OPS. (Febrero de 2020). Organización Panamericana de la Salud - OPS. Recuperado el 02 de Enero de 2021, de https://www.paho.org/hq/index.php?option=com_content\&view=article\&id=15716:country-cancer-profiles-2020\&Itemid $=72576 \&$ lan$\mathrm{g}=\mathrm{es} \#: \sim$ :text=El\%20c\%C3\%A1ncer\%20es\%20 la\%20segunda,colorrectal\%20(8\%2C0\%25).

Sociedad Americana Contra el Cáncer. (30 de Marzo de 2020). Sociedad Americana Contra el Cáncer. Recuperado el 15 de Enero de 2021, de https:// www.cancer.org/es/cancer/cancer-de-esofago/ deteccion-diagnostico-clasificacion-por-etapas/ clasificacion-por-etapas.html

Sociedad Americana Contra el Cáncer. (20 de Enero de 2020). Sociedad Americana Contra el Cáncer. Recuperado el 28 de Enero de 2021, de https:// www.cancer.org/es/cancer/cancer-de-esofago/ tratamiento/por-etapa.html

Sociedad Americana Contra el Cáncer. (12 de Enero de 2021). Sociedad Americana Contra el Cáncer. Recuperado el 13 de Enero de 2021, de https:// www.cancer.org/es/cancer/cancer-de-esofago/ acerca/estadisticas-clave.html

Téllez, O., \& Hodelín, D. (2016). Cáncer de esófago. Una actualización necesaria. Morfovirtual, 1-15. Recuperado el 20 de Enero de 2021, de http:// www.morfovirtual2016.sld.cu/index.php/Morfovirtual/2016/paper/download/368/203

\section{CITAR ESTE ARTICULO:}

Zambrano Zambrano, F. C., Vélez Macías, M. A., Chacha Suscal, N. R., Basurto Macias, G. G., \& Pesantez Durán, F. A. (2020). Sintomatología y tratamiento en cada etapa del paciente con cáncer de esófago. RECIAMUC, 4(4), 263-

272. https://doi.org/10.26820/reciamuc/4.(4).noviembre.2020.263-272 